\title{
Comparación entre ancianos sanos con alta y baja reserva cognitiva y ancianos con deterioro cognitivo*
}

\section{Comparison between Healthy Elderly with High and Low Cognitive Reserve and Cognitive Impairment Elderly}

Recibido: octubre 22 de 2011 | Revisado: marzo 3 de 2012 | Aceptado: junio 10 de 2012

\author{
JuAN CARLOS MELÉNDEZ MORAL ** \\ Teresa MAYORdOMO RODRÍGUEZ \\ Alicia SAlEs GaláN *** \\ Universidad de Valencia, España
}

SICI: 1657-9267(201303)12:1<73:CABRCD>2.0.TX;2-8

Para citar este artículo: Meléndez, J. C., Mayordomo, T. \& Sales, A. (2013). Comparación entre ancianos sanos con alta y baja reserva cognitiva y ancianos con deterioro cognitivo. Universitas Psychologica, 12(1), 73-80.

* Investigación realizada con el apoyo del Ministerio Español de Ciencia e Innovación bajo el proyecto PSI2010-17425.

** Departamento de Psicología Evolutiva y de la Educación.Facultad de Psicología. E-mails: melendez@ uv.es, temaro2@alumni.uv.es

**** Departamento de Metodología de las Ciencias del Comportamiento. Facultad de Psicología. E-mail: Alicia.Sales@uv.es

\section{RES UMEN}

Este trabajo presenta la comparación de las características de sujetos con alta y baja reserva cognitiva, y sujetos con baja reserva cognitiva y deterioro cognitivo, diferenciados a partir del desarrollo de una medida ponderada de reserva cognitiva. Mediante un diseño correlacional se evaluaron 200 personas con edad superior a 65 años, no institucionalizados. Los resultados mostraron, por un lado, que el grupo de alta reserva cognitiva obtuvo mejores puntuaciones tanto en el nivel de deterioro cognitivo como en la medida ponderada de reserva que el grupo de baja reserva cognitiva y, por otro lado, el grupo de baja reserva cognitiva mostró un perfil similar al del grupo de deterioro cognitivo, existiendo solamente diferencias en cuanto a la edad. Las diferencias observadas permiten plantear la medida como una variable que se debe tener en cuenta en el diagnóstico de enfermedades neurodegenerativas y su utilidad para establecer posibles tratamientos.

Palabras clave autores

Reserva cognitiva, deterioro cognitivo leve, ancianos sanos, compensación.

Palabras clave descriptores

Psicología del desarrollo, psicología cognitiva, investigación cuantitativa.

\footnotetext{
A B S T R A C T

This paper presents the comparison of the characteristics of subjects with high and low cognitive reserve and subjects with low cognitive reserve and cognitive impairment, differentiated from the development of a weighted measure of cognitive reserve. Using a correlational design was evaluated 200 people aged over 65 , not institutionalized. The results showed, firstly that high cognitive reserve group performed better on both the level of cognitive impairment as the weighted average of reserves that the group of low cognitive reserve and on the other hand the low cognitive reserve group showed a similar profile to the group of cognitive impairment, existing differences only in age. The observed differences would support the measure as a variable to be considered in the diagnosis of neurodegenerative diseases, and its usefulness to determine possible treatments.

Key words authors

Cognitive reserve, mild cognitive impairment, elderly healthy, compensation.

Key words plus

Development Psychology, Cognitive Psychology, Quantitative Research.
} 


\section{Introducción}

Con la edad, existen pérdidas en las funciones cognitivas que en algunos casos se convierten en patológicas, produciéndose deterioro cognitivo de diversa índole y características. Una de las variables que puede determinar la velocidad con la que se producen estos cambios ha sido definida como reserva.

La reserva, según Stern (2009), es la capacidad que tienen determinadas personas para demorar el deterioro cognitivo que acaece con el envejecimiento, manteniéndose, según Rami et al. (2011), clínicamente poco afectadas a pesar de que los datos de neuroimagen o neuropatológicos muestren un proceso avanzado de la enfermedad. Así, se puede entender como una mayor probabilidad para tolerar los efectos de la patología asociada al deterioro, es decir, para soportar mayor cantidad de neuropatología antes de llegar al umbral donde la sintomatología clínica comienza a manifestarse (Manly, Schupf, Tang, Weissm \& Stern, 2007; Stern, 2002, 2003), habiéndose distinguido para su operativización dos perspectivas complementarias: el modelo pasivo y el activo.

El modelo pasivo ha sido definido como reserva cerebral (Katzman, 1993) o reserva neural (Mortimer, Schuman \& French, 1981), haciendo referencia su operacionalización al tamaño del cerebro, número de neuronas o densidad sináptica. Los modelos activos sostienen la existencia de una intencionalidad para minimizar los efectos del deterioro, pudiéndose poner en marcha los procesos de reserva cognitiva (RC) y compensación, definida esta última como el uso de estrategias o mecanismos que ayudan a superar o mitigar la disminución del funcionamiento de la memoria (Backman \& Dixon, 1992; Frias, Dixon \& Bäckman, 2003), diferenciándose dos tipos de estrategias básicas: las implícitas (estructuras cerebrales) y las explícitas (estrategias cognitivas y conductuales) (Díaz, Buiza \& Yanguas, 2009; Meléndez, Mayordomo, Sales, Cantero \& Viguer, 2013).

Según la investigación científica desarrollada desde los modelos activos de RC, existen múltiples variables que influyen en su desarrollo, mantenimiento y/o potenciación a lo largo del ciclo vital.
Aunque no existen estudios concluyentes en relación con la relevancia de cada uno de los componentes, así como la combinación más adecuada de la medida, para su operacionalización se han utilizado variables como educación, ocupación, nivel de inteligencia premórbida, y actividades de ocio, físicas, mentales y sociales han sido las más empleadas, obteniendo resultados concluyentes (Bosch et al., 2010; Corral, Rodriguez, Amenedo, Sanchez \& Diaz, 2006; León, García \& RoldánTapia, 2011; Rami et al., 2011; Solé-Padullés et al., 2009; Stern, 2009; Stern et al., 2005; Valenzuela \& Sachdev, 2006). Así, diferentes estudios (Bosch et al., 2010; León et al., 2011; Solé-Padullés et al., 2009; Stern, 2009; Stern et al., 2005) presentan mediante análisis factorial exploratorio medidas de RC combinadas, a partir de las cuales analizan su relación con diferentes patologías cognitivas.

En este sentido, uno de los retos de la presente investigación es comparar grupos de ancianos sanos con diferentes niveles de RC, así como analizar las similitudes y diferencias con ancianos con deterioro cognitivo, puesto que el estudio de las variables que se asocian a la formación de la RC son de gran relevancia para el diagnóstico de diversas enfermedades como la EA en fase preclínica, dado que la RC podría modificar el curso de la enfermedad (Stern, 2006).

De este modo, en este trabajo se desarrolló una medida de RC para población española, siguiendo el procedimiento descrito por autores como Scarmeas (2007); Scarmeas et al. (2003); Solé-Padullés et al. (2009), Stern (2009), Stern et al. (2005). A partir de la obtención de la medida de RC se compararon sujetos con alta reserva cognitiva (ARC) y baja reserva cognitiva (BRC), así como sujetos con BRC y deterioro cognitivo, planteándose como hipótesis para poner a prueba:

Hipótesis 1: al comparar sujetos con ARC y BRC no se observarán diferencias significativas en las variables sociodemográficas estudiadas.

Hipótesis 2: al comparar sujetos con ARC y BRC se obtendrán diferencias significativas en las puntuaciones obtenidas en la medida ponderada de RC y en el Mini-Mental State Examination (MMSE). 
Hipótesis 3: al comparar sujetos con BRC y deterioro cognitivo se obtendrán diferencias significativas en las variables sociodemográficas estudiadas.

Hipótesis 4: al comparar sujetos con BRC y deterioro cognitivo se obtendrán diferencias significativas en las puntuaciones obtenidas en la medida ponderada de $\mathrm{RC}$ y en el MMSE.

Hipótesis 5: en los grupos de ARC y BRC las puntuaciones de la medida ponderada de $\mathrm{RC}$ y MMSE mostrarán relaciones positivas y significativas, mientras que para la edad serán negativas y significativas.

Hipótesis 6: en los grupos de BRC y deterioro cognitivo las puntuaciones de la medida ponderada de RC y MMSE mostrarán relaciones positivas y significativas con la edad.

\section{Métodos}

\section{Muestra}

El diseño de investigación es correlacional, habiéndose diferenciado dos grupos: sanos y con deterioro cognitivo leve (DCL). El grupo de sujetos sanos está compuesto por 178 sujetos con una edad media de $74.37(\mathrm{DE}=6.9)$ de los cuales el $56.2 \%$ son mujeres, estableciéndose como criterios de inclusión: tener edad igual o superior a 65 años y no tener diagnóstico o antecedentes de enfermedad neurológica ni psiquiátrica. El grupo DCL está compuesto por 23 sujetos con una edad media de $80.3(D E=5.8)$ de los cuales el $82.6 \%$ son mujeres, que fueron diagnosticados desde el servicio de neurología de la Agencia Valenciana de Salud, estableciéndose como criterios para su inclusión la obtención de una puntuación inferior a 23 en el Mini-Mental State Examination (MMSE), un nivel 2-3 en la escala GDS y haberse desestimado el diagnóstico de demencia o enfermedades psiquiátricas.

\section{Instrumentos}

Siempre de forma individual, los participantes respondieron, en primer lugar, un cuestionario de datos sociodemográficos. A continuación se aplicó el MMSE (Lobo, Saz \& Marcos, 2002) y la subprueba de vocabulario de la Escala de Inteligencia Wechsler para Adultos-III (WAIS-III; Wechsler, 2001) para analizar el nivel de inteligencia premórbida. Finalmente, se indagó si en los últimos años realizaban o no una serie de 14 actividades de ocio y estimulantes (pasatiempos, leer, escribir, bailar, ejercicio, deporte, manualidades, estudio/talleres, instrumento, voluntariado, cine, juegos de mesa, pertenencia a asociaciones culturales y viajar).

Para el desarrollo de la medida de RC, tal y como se mencionó anteriormente, se siguieron las pautas establecidas en otros trabajos (Scarmeas, 2007; Scarmeas et al., 2003; Solé-Padullés et al., 2009; Stern, 2009; Stern et al., 2005), donde se plantean diferentes variables-medida que están relacionadas directamente con la RC. De este modo, se incluyó el WAIS-III, subprueba vocabulario, aplicándose los baremos de conversión en función de la edad, con un rango de puntuaciones entre 1 y 19. Una segunda medida, definida como educación-ocupación, fue codificada usando valores ordinales que en el caso de educación fueron: 1 = no educación formal, $2=$ primaria, $3=$ secundaria, $4=$ universitaria; y en ocupación: 1 = manual no cualificado, $2=$ manual cualificado, $3=$ no manual cualificado o técnico, 4 = profesional (título universitario requerido), $5=$ manager o director. El valor final se obtuvo sumando los valores educativos y ocupacionales (rango 2-9). Como tercera medida se tuvieron en cuenta actividades de ocio que fueran cognitivamente estimulantes (Scarmeas \& Stern, 2003), que implicaran algún tipo de actividad física y que tuviesen en cuenta la participación social o en grupo evaluadas por los sujetos mediante una respuesta dicotómica de si la realizaban o no y estableciéndose un rango de puntuaciones entre 0 y 14 .

Para conocer el peso factorial de estas tres medidas, se realizó un análisis factorial exploratorio con método de componentes principales (Solé-Padullés et al., 2009; Stern et al., 2005) a partir de los sujetos sanos, obteniendo una varianza explicada del $53.3 \%$. Las saturaciones factoriales de cada variable fueron multiplicadas por la puntuación de cada sujeto en cada variable obteniéndose, mediante su sumatoria, una medida combinada de RC. En la 
Tabla 1 se pueden observar los principales datos de las variables.

\section{TABLA 1}

Medias, desviaciones típicas, correlación ítem-factor y saturación factorial de las variables

\begin{tabular}{lllcc}
\hline & M & DE & ri-f & 1 \\
\hline WAIS-III vocabulario & 11.1 & 2.4 & $0.586 * *$ & 0.560 \\
Estudio-Ocupación & 4.1 & 2.0 & $0.777 * *$ & 0.808 \\
Actividades & 4.3 & 2.3 & $0.769 * *$ & 0.757 \\
\hline
\end{tabular}

Nota. La correlación es significativa al nivel 0.01 .

Fuente: elaboración propia.

\section{Análisis}

En primer lugar se realizó un análisis factorial exploratorio para la obtención de la medida ponderada de reserva cognitiva. Para el análisis de las diferencias entre los grupos se utilizó la $U$ de Mann-Whitney, chi cuadrado y pruebas $t$ para muestras independientes dependiendo del tipo de variable. Además se aplicaron correlaciones de Pearson. Los análisis estadísticos se realizaron mediante SPSS 19.

\section{TABLA 2}

Media y desviación típica y distribución de participantes de las variables para los grupos de baja y alta reserva cognitiva

\begin{tabular}{lccc}
\hline & BRC & ARC & Sig. \\
\hline Edad & $76.41(7.1)$ & $72.39(6.2)$ & 0.006 \\
Genero & & & \\
Hombre & 36.4 & 61.4 & 0.019 \\
Mujer & 63.6 & 38.6 & \\
Estado civil & & & \\
$\begin{array}{l}\text { Casado } \\
\text { Soltero }\end{array}$ & 52.3 & 72.7 & n.s. \\
Viudo & 4.5 & 6.8 & \\
Educación & 43.2 & 20.5 & \\
Sin estudios & & & \\
$\begin{array}{l}\text { Primarios } \\
\text { Secundarios }\end{array}$ & 45.5 & 6.8 & \\
Superiores & 52.3 & 13.6 & 0.000 \\
$\begin{array}{l}\text { Profesión } \\
\text { Manual no cualificado }\end{array}$ & 7.3 & 25 & \\
Manual cualificado & 75 & 13.6 & \\
No manual cualificado & 25 & 27.3 & 0.000 \\
Profesional con titulo & & 2.3 & \\
Directivos & & 40.9 & \\
Actividades & & 15.9 & \\
\hline
\end{tabular}

Fuente: elaboración propia.

\section{Resultados}

Partiendo de la medida de RC se diferenciaron dos grupos, uno compuesto por aquellos sujetos situados por encima del centil $75(N=44)$, que fueron definidos como grupo de alta reserva cognitiva (ARC) y otro compuesto por aquellos sujetos situados por debajo del centil $25(N=44)$, definidos como grupo de baja reserva cognitiva (BRC), desestimándose un total de 90 sujetos que ocupaban la posición central.

A partir de esta diferenciación, se realizó el estudio de las dos primeras hipótesis planteadas en las que se pretendía comparar las características de los ancianos sanos asignados a los grupos de reserva cognitiva. De este modo, al comparar los sujetos con alta y baja reserva cognitiva en sus diferentes variables sociodemográficas, se obtuvieron diferencias significativas en todas las variables estudiadas a excepción del estado civil, tal y como se observa en la Tabla 2.

Además, se comprobó la existencia de diferencias significativas para la medida de RC ponderada $(t(86)=27.1 ; p<0.001)$, observándose que la

\section{TABLA 3}

Media y desviación típica y distribución de participantes de las variables para los grupos de baja cognitiva y deterioro cognitivo

\begin{tabular}{lccc}
\hline & BRC & DCL & Sig. \\
\hline Edad & $76.41(7.1)$ & $80.3(5.8)$ & 0.028 \\
Genero & & & \\
Hombre & 36.4 & 17.4 & n. s. \\
$\begin{array}{l}\text { Mujer } \\
\text { Estado civil }\end{array}$ & 63.6 & 82.6 & \\
$\begin{array}{l}\text { Casado } \\
\text { Soltero }\end{array}$ & 52.3 & 30.4 & \\
Viudo & 4.5 & & n. s. \\
$\begin{array}{l}\text { Educación } \\
\text { Sin estudios }\end{array}$ & 43.2 & 69.6 & \\
$\begin{array}{l}\text { Primarios } \\
\text { Secundarios }\end{array}$ & 45.5 & 60.9 & \\
$\begin{array}{l}\text { Superiores } \\
\text { Profesión }\end{array}$ & 2.3 & 30.4 & n. s. \\
$\begin{array}{l}\text { Manual no cualificado } \\
\text { Manual cualificado }\end{array}$ & 75 & 8.7 & \\
$\begin{array}{l}\text { No manual cualificado } \\
\text { Profesional con titulo }\end{array}$ & 25 & 8.6 & \\
$\begin{array}{l}\text { Directivos } \\
\text { Actividades }\end{array}$ & & 8.7 & n.s. \\
\hline
\end{tabular}

Fuente: elaboración propia. 
media de los sujetos de ARC (17.6; DE $=1.9)$ era superior a los de BRC (8.9; DE = 0.9). Del mismo modo, se obtuvieron diferencias significativas entre los grupos en la puntuación del MMSE $(t(86)=$ 4.07; $p<0.001)$, siendo la media de los sujetos de $\operatorname{ARC}(28.5 ; D E=1.7)$ superior a la de los sujetos de $\operatorname{BRC}(26.9 ; D E=1.9)$.

A continuación, se pusieron a prueba las hipótesis tres y cuatro para el análisis de los grupos de $\mathrm{BRC}$ y deterioro cognitivo. En primer lugar, al comparar las variables sociodemográficas de los grupos de BRC y deterioro cognitivo, no se obtuvieron diferencias significativas exceptuando la edad, tal y como se observa en la Tabla 3.

Por lo que respecta a la cuarta hipótesis, la comparación de las puntuaciones obtenidas en la medida ponderada de RC y en el MMSE, para los grupos de BRC y deterioro cognitivo, solamente mostró resultados significativos en la puntuación del MMSE $\left(F_{(1,65)}=3.94, p<0.001\right)$, siendo la media de los sujetos de BRC (26.91) superior a los de DCL (18.30).

Finalmente, para las hipótesis quinta y sexta, en las que se pretendía estudiar la relación entre la medida combinada de RC, MMSE y la edad, se observaron relaciones significativas entre todas las variables en el grupo de sanos. De este modo, la edad mostró una relación significativa y negativa con la puntuación del MMSE $(r=-0.410 ; p<0.001)$, y con la puntuación combinada de $\mathrm{RC}(r=-0.206$; $p<0.000$ ) y el MMSE obtuvo una relación significativa y positiva con la puntuación combinada de $\mathrm{RC}(r=0.316 ; p<0.001)$. Por contra, el estudio de las relaciones entre las medidas en los grupos de BRC y deterioro cognitivo, no mostró ningún tipo de relación significativa.

\section{Discusión}

Este trabajo ofrece resultados en relación con las diferencias entre sujetos con alta y baja reserva cognitiva, así como la comparación de los sujetos con baja reserva cognitiva y deterioro cognitivo. De este modo, se ha podido comprobar cómo mientras los grupos de alta y baja reserva eran significativamente distintos, la comparación del grupo de baja reserva cognitiva y deterioro cognitivo ha mostrado importantes similitudes.

Por otra parte, y de forma previa al análisis y comparación de los grupos, se ha presentado un procedimiento para el desarrollo de la medida ponderada de RC en una muestra de ancianos sanos. Existe acuerdo entre los diferentes investigadores en que los criterios que se deben utilizar deben estar relacionados con las habilidades cognitivas, la educación, la ocupación laboral, las actividades de ocio mentalmente estimulantes y la inteligencia premórbida, pudiendo ser utilizados como predictores de la demencia con un alto grado de consistencia empírica (Valenzuela \& Sachdev, 2006). En este sentido, se han incluido diferentes criterios que hasta ahora eran utilizados mayoritariamente de forma independiente en la literatura científica, habiéndose obtenido una medida con adecuadas propiedades psicométricas a través del análisis factorial exploratorio.

De forma más concreta y por lo que respecta a los criterios utilizados en la obtención de la medida, ha sido la variable educación uno de los principales predictores, habiéndose observado que aquellos sujetos con altos niveles educativos mantienen una mejor función cognitiva y un menor riesgo de pérdidas y de síntomas de demencia con la edad (Vance \& Crowe, 2006). Además, la educación parece promover la formación de conexiones neuronales pudiendo a su vez representar una mayor propensión a participar en actividades mentalmente estimulantes. En relación con la ocupación, según Stern (2006), cuando es utilizada individualmente parece no predecir la aparición de síntomas de demencia y cuando lo hace es asociada a la educación.

En relación con las actividades de tiempo libre y mentalmente estimulantes, el trabajo de Valenzuela y Sachdev (2006) examinó su relación con la incidencia ante el deterioro cognitivo, informando de un claro efecto protector después de controlar por un número relevante de covariadas, conclusiones paralelas a otros trabajos, según los cuales existe clara evidencia empírica de sus beneficios para el envejecimiento en general y para el retraso de la EA (Scarmeas \& Stern, 2003; Vance \& Crowe, 2006). De forma más específica, al estudiar la relación en- 
tre las actividades de tiempo libre y la cognición, Richards, Hardy y Wadsworth (2003) observaron que la realización de las actividades está positivamente relacionada con la función cognitiva después de los 43 años. Por otra parte, uno de los claros efectos de este criterio es el aumento del flujo sanguíneo, así como la generación de una mayor plasticidad cerebral (Richards \& Deary, 2005).

Finalmente, y por lo que se refiere a la inteligencia premórbida Solé-Padullés et al. (2009) informan de la existencia de una clara relación entre la materia gris y este criterio. En esta misma línea de resultados, Colom, Jung y Haier (2006) obtuvieron evidencia de la relación positiva entre las medidas del WAIS, incluida la subprueba de vocabulario, y el incremento del volumen de la materia gris.

Tal y como se ha podido observar, la medida conjunta de educación y ocupación ha obtenido una alta correlación con la medida de reserva cognitiva, siendo además el elemento con mayor saturación factorial. También las actividades han mostrado una alta relación con la medida de reserva cognitiva, así como una importante saturación factorial en la medida, confirmando su adecuación como variable en la evaluación cognitiva de las personas ancianas y en el estudio de los posibles factores protectores frente al deterioro cognitivo. Por lo que respecta a la estimación de la inteligencia premórbida, aun siendo una variable a tener en cuenta, cabe destacar que ha sido la variable que ha obtenido la menor de las saturaciones factoriales, siendo por tanto, tal y como plantean el trabajo de Stern (2006), los criterios educación y ocupación y el criterio actividades, los que muestran tanto de forma individual como de forma conjunta un mayor efecto sobre la medida de RC.

En relación a las hipótesis, en primer lugar se pusieron a prueba aquellas en las que se encontraban los grupos con ARC y BRC. La primera de las hipótesis en la que se planteaba que no existirían diferencias significativas en las variables sociodemográficas estudiadas, no se cumplió dado que se observaron diferencias en todas las variables estudiadas exceptuando el estado civil. Por lo que respecta a las diferencias encontradas, se ha podido comprobar la existencia de un mayor porcentaje de hombres en el grupo de ARC, mientras que en el grupo de BRC son las mujeres las que mayoritariamente lo componen. En relación con las demás variables estudiadas: nivel de educación, ocupación, actividades y WAIS III vocabulario, las puntuaciones más altas las han obtenido los sujetos con ARC, siendo los resultados similares a los hallados por Stern et al. (2005). Por lo que respecta a la edad también se observaron diferencias entre los grupos, siendo superior la media de edad del grupo BRC. En cuanto a la segunda hipótesis, cabe señalar las diferencias significativas encontradas tanto en la puntuación obtenida en la medida de RC como las obtenidas en la medida de deterioro cognitivo leve, señalando que los sujetos pertenecientes al grupo de ARC podrían beneficiarse en mayor medida de los ventajas producidos por la RC, y mantener las capacidades cognitivas por encima de los sujetos de BRC, confirmando por tanto la hipótesis de partida.

Las hipótesis tercera y cuarta, que ponían a prueba la existencia de diferencias entre los grupos de BRC y deterioro cognitivo, no se han cumplido dado que no se observaron diferencias significativas -exceptuando la edad- para ninguna variable sociodemográficas, como tampoco para la medida ponderada de RC. De este modo, los grupos estudiados mostraron dos perfiles similares de puntuaciones, siendo destacables dos aspectos: la diferencia de edad y la no diferencia de la medida ponderada de RC. Con relación a las diferencias en edad, en las que el grupo de deterioro tiene una media superior, debe tenerse en cuenta que probablemente vienen dadas por la alta relación de la edad con este tipo de patologías, efecto indicado por Corral et al. (2006), pero, por otra parte, el aumento de edad del grupo con BRC anularía las diferencias entre los grupos. Además, la no existencia de diferencias entre ambos grupos para la medida ponderada de reserva cognitiva, implica que el efecto protector asignado a la $\mathrm{RC}$ sea semejante en los sujetos con BRC y con deterioro cognitivo. De este modo, existe una más alta probabilidad de conversión de sujetos con BRC a sujetos con deterioro cognitivo.

Respecto a las relaciones entre las medidas, la quinta hipótesis se ha cumplido, habiéndose observado relaciones significativas entre la medida de 
RC, el MMSE y la edad para los grupos de ARB y BRC. La edad ha mostrado correlaciones negativas con la medida ponderada de RC y con el MMSE; es decir, a más edad más posibilidad de que se produzca deterioro y menor capacidad de $\mathrm{RC}$, resultados similares a los obtenidos por Corral et al. (2006) que señalan que la $\mathrm{RC}$ es un factor protector relacionado con la edad. Finalmente y en relación con la sexta hipótesis por desarrollar, no se ha podido cumplir, ya que para los grupos de BRC y DCL no existen relaciones significativas en ninguna de las variables estudiadas.

Por último, se puede afirmar partiendo de la literatura científica sobre el modelo activo de $\mathrm{RC}$ a través de la cual se ha obtenido una medida ponderada de $\mathrm{RC}$, que se puede realizar el análisis de las características que poseen tanto los ancianos sanos con ARC o BRC así como sujetos con deterioro cognitivo, siendo dichos hallazgos de gran utilidad para el diagnóstico de posibles enfermedades neurodegenerativas así como para la personalización de los posibles tratamientos que se deben realizar. Además, y a partir de los resultados obtenidos en este trabajo, se plantea como línea futura de investigación, el análisis y conocimiento de cuáles son las estrategias compensatorias más utilizadas en aquellos sujetos con alta RC, de forma que en la aplicación de tratamientos no farmacológicos pudieran ser incluidas.

\section{Referencias}

Backman, L. \& Dixon, R. A. (1992). Psychological compensation: A theoretical framework. Psychological Bulletin, 112(2), 259-283.

Bosch, B., Bartrés-Faz, D., Rami, L., Arenaza-Urquijo, E., Fernández-Espejo, D., Junqué, C., et al. (2010). Cognitive reserve modulates task-induced activations and deactivations in healthy elders, amnestic mild cognitive impairment and mild Alzheimer's disease. Cortex, 46(4), 451-461.

Colom, R., Jung, R. E. \& Haier, R. J. (2006). Distributed brain sites for the g-factor intelligence. Neuroimage, 31(3), 1359-1365.

Corral, M., Rodriguez, M., Amenedo, E., Sanchez, J. L. \& Diaz, F. (2006). Cognitive reserve, age, and neuropsychological performance in healthy participants. Developmental Neuropsychology, 29(3), 479-491.

Díaz, U., Buiza, C. \& Yanguas, J. (2009). Reserva cognitiva: evidencias, limitaciones y líneas de investigación futura. Revista Española de Geriatría y Gerontología, 45(3), 150-155.

Frias, C. M., Dixon, R. A. \& Bäckman, L. (2003). Use of memory compensation strategies is related to psychosocial and health indicators. Journals of Gerontology Series B: Psychological Sciences and Social Sciences, 58(1), 12-22.

Katzman, R. (1993). Education and the prevalence of dementia and Alzheimer's disease. Neurology, 43(1), 13-20.

León, I., García, J. \& Roldán-Tapia, L. (2011). Construcción de la escala de reserva cognitiva en población española: estudio piloto. Revista de Neurología, 52(11), 653-660.

Lobo, A., Saz, P. \& Marcos, G. (2002). Adaptación del Examen Cognoscitivo Mini-Metal. Madrid: Tea Ediciones.

Manly, J. J., Schupf, N., Tang, M. X., Weissm, C. C. \& Stern, Y. (2007). Literacy and cognitive decline among ethnically diverse elders. En Y. Stern (Ed.), Cognitive reserve theory and applications (pp. 219. 236). New York: Taylor \& Francis.

Melendez, J. C, Mayordomo, T. Sales, A., Cantero, M. J. $\&$ Viguer, P. (2013). How we compensate for memory loss in old age: Adapting and validating the Memory Compensation Questionnaire (MCQ) for Spanish population. Archives of Gerontology and Geriatrics, 56(1), 32-37.

Mortimer, J. A., Schuman, L. \& French, L. (1981). Epidemiology of dementing illness. En J. A. Mortimer \& L. M. Schuman (Eds.), The epidemiology of dementia: Monographs in epidemiology and biostatistics (pp. 323-333). New York: Oxford University Press.

Rami, L., Valls-Pedret, C., Bartrés-Faz, D., Caprile, C., Solé-Padullés, C., Castellví, M., et al. (2011). Cuestionario de reserva cognitiva. Valores obtenidos en población anciana sana y con enfermedad de Alzheimer. Revista de Neurología, 52(4), 195-201.

Richards, M. \& Deary, I. J. (2005). A life course approach to cognitive reserve: A model for cogniti- 
ve aging and development? Annals of Neurology, 58(4), 617-622.

Richards, M., Hardy, R. \& Wadsworth, M. E. J. (2003). Does active leisure protect cognition? Evidence from a national birth cohort. Social Science \& Medicine, 56(4), 785-792.

Scarmeas, N. (2007). Life style patterns and cognitive reserve. En Y. Stern (Ed.), Cognitive reserve: Theory and applications (pp. 187-206). New York: Taylor $\&$ Francis.

Scarmeas, N. \& Stern, Y. (2003). Cognitive reserve and lifestyle. Journal of Clinical and Experimental Neuropsychology, 25(5), 625-633.

Scarmeas, N., Zarahn, E., Anderson, K. E., Hilton, J., Flynn, J., Van Heertum, R. L., et al. (2003). Cognitive reserve modulates functional brain responses during memory tasks: A PET study in healthy young and elderly subjects. Neuroimage, 19(3), 1215-1227.

Solé-Padullés, C., Bartrés-Faz, D., Junqué, C., Vendrell, P., Rami, L., Clemente, I., et al. (2009). Brain structure and function related to cognitive reserve variables in normal aging, mild cognitive impairment and Alzheimer's disease. Neurobiology of Aging, 30(7), 1114-1124.
Stern, Y. (2002). What is cognitive reserve? Theory and research application of the reserve concept. Journal of the International Neuropsychological Society, 8(3), 448-460.

Stern, Y. (2003). The concept of cognitive reserve: A catalyst for research. Journal of Clinical and Experimental Neuropsychology, 25(5), 589-593.

Stern, Y. (2006). Cognitive reserve and Alzheimer Disease. Alzheimer Disease and Associated Disorders, 20(2), 112-117.

Stern, Y. (2009). Cognitive reserve. Neuropsychologia, 47(10), 2015-2028.

Stern, Y., Habeck, C., Moeller, J., Scarmeas, N., Anderson, K. E., Hilton, H. J., et al. (2005). Brain networks associated with cognitive reserve in healthy young and old adults. Cerebral Cortex, 15(4), 394-402.

Valenzuela, M. \& Sachdev, P. (2006). Brain reserve and dementia: A systematic review. Psychological Medicine, 36(4), 441-454.

Vance, D. E. \& Crowe, M. (2006). A proposed model of neuroplasticity and cognitive reserve in older adults. Activities, Adaptation \& Aging, 30(3), 61-79.

Wechsler, D. (2001). Wechsler Intelligence Scale for Adults-III (WAIS-III). Madrid: TEA Ediciones. 